\title{
Heightened activity in social reward networks is associated with adolescents' risky sexual behaviors
}

\author{
Kristen L. Eckstrand ${ }^{\mathrm{a}}$, Sophia Choukas-Bradley ${ }^{\mathrm{b}}$, Arpita Mohanty ${ }^{\mathrm{b}}$, Marissa Cross ${ }^{\mathrm{b}}$, \\ Nicholas B. Allen ${ }^{c}$, Jennifer S. Silk ${ }^{\mathrm{d}}$, Neil P. Jones ${ }^{\mathrm{b}}$, Erika E. Forbes ${ }^{\mathrm{e} \text {, }}$ \\ a Western Psychiatric Institute and Clinic, Department of Psychiatry, University of Pittsburgh, Pittsburgh, PA, United States \\ ${ }^{\mathrm{b}}$ Department of Psychiatry, University of Pittsburgh, Pittsburgh, PA, United States \\ c Department of Psychology, University of Oregon, Eugene, OR, United States \\ d Departments of Psychology and Psychiatry, University of Pittsburgh, Pittsburgh, PA, United States \\ e Departments of Psychiatry, Psychology, and Pediatrics, University of Pittsburgh, Pittsburgh, PA, United States
}

\section{A R T I C L E I N F O}

\section{Keywords:}

Sexual risk

Sexual behavior

Adolescence

Social reward

Peers

Affective neuroscience

\begin{abstract}
A B S T R A C T
Adolescent sexual risk behavior can lead to serious health consequences, yet few investigations have addressed its neurodevelopmental mechanisms. Social neurocircuitry is postulated to underlie the development of risky sexual behavior, and response to social reward may be especially relevant. Typically developing adolescents $(N=47 ; 18 \mathrm{M}$, 29F; $16.3 \pm 1.4$ years; $42.5 \%$ sexual intercourse experience) completed a social reward fMRI task and reported their sexual risk behaviors (e.g., lifetime sexual partners) on the Youth Risk Behavior Survey (YRBS). Neural response and functional connectivity to social reward were compared for adolescents with higher- and lower-risk sexual behavior. Adolescents with higher-risk sexual behaviors demonstrated increased activation in the right precuneus and the right temporoparietal junction during receipt of social reward. Adolescents with higher-risk sexual behaviors also demonstrated greater functional connectivity between the precuneus and the temporoparietal junction bilaterally, dorsal medial prefrontal cortex, and left anterior insula/ ventrolateral prefrontal cortex. The greater activation and functional connectivity in self-referential, social reward, and affective processing regions among higher sexual risk adolescents underscores the importance of social influence underlying sexual risk behaviors. Furthermore, results suggest an orientation towards and sensitivity to social rewards among youth engaging in higher-risk sexual behavior, perhaps as a consequence of or vulnerability to such behavior.
\end{abstract}

\section{Introduction}

Compared with both adults and children, adolescents have an enhanced propensity to engage in rewarding, risky behavior (Somerville et al., 2010). Sexual risk behavior - including early sexual debut, higher number of partners, or unprotected intercourse - is a particularly important domain of risk behavior that emerges during adolescence, as it involves decisions to engage in potentially highly rewarding experiences that can lead to serious health consequences, including HIV and other sexually transmitted infections (STIs), or unplanned pregnancy (Abma et al., 2010; Kann, 2016).

Changes in risky behavior occur in tandem with greater behavioral and neural sensitivity to rewards during adolescence, the transitional developmental period between the completion of puberty and the attainment of adulthood status (Casey et al., 2008; Somerville et al., 2010). Conceptual models of the rise in sensation seeking during adolescence have focused on the balance among the brain's affective and control systems, positing a more protracted developmental course in control systems than reward systems (Casey et al., 2008; Somerville et al., 2010; Steinberg, 2005) or a temporary imbalance of influence among threat, reward, and control systems (Ernst and Fudge, 2009). In addition, development in the dopamine system over the course of adolescence leads to greater innervation of medial prefrontal cortex (mPFC), with implications for enhanced capacity for inhibitory control generally and regulation of basic striatal reward responding (Gomes et al., 2016; Luna et al., 2015). Models of adolescent neurodevelopment have recently been extended to adolescents' sexual behavior (Victor and Hariri, 2016), with the hypothesis that increased reward responsiveness, decreased threat detection and reactivity, and immature behavioral regulation capacity underlie risky sexual behavior.

Social context and the evaluation of social information relative to oneself (e.g. self-referential processing) are critical factors in adolescent

\footnotetext{
* Corresponding author.

E-mail address: forbesee@upmc.edu (E.E. Forbes).
} 
brain and sexual development. During adolescence, the aforementioned changes in the neurodevelopmental balance of threat, reward, and control systems co-occurs with a heightened sensitivity to social stimuli, in which youth are highly motivated to affiliate and gain status with peers (Albert et al., 2013; Crone and Dahl, 2012). Further, the neural circuitry underlying self-referential processing undergoes maturation during adolescence, with particular implications for social behavior, including sexual behavior (Sebastian et al., 2008). Specifically, adolescents are increasingly aware of others' evaluation of them and that they may change their behavior in order to receive more positive evaluation. Indeed, adolescents' engagement in risky behaviors is often met with peer rewards, such as increased social status (Brechwald and Prinstein, 2011). It is thus unsurprising that most of adolescents' risky behaviors occur in peer social contexts (Albert et al., 2013). Social context-while relevant for a broad range of adolescent risk behaviors-may be especially salient in sexual risk behavior. Sexual behavior is inherently interpersonal, and is unique among health risk behaviors because it involves high emotional arousal in response to both environmental and interoceptive stimuli (Victor and Hariri, 2016). During adolescence, sexual activity is a novel social experience that can have a variety of meanings including status changes among peers (e.g., Mayeux et al., 2008) and the formation of intimate romantic relationships (e.g., Williams and Russell, 2013).

The integration of the brain's social and self-referential circuitry with key regions including precuneus and temporoparietal junction (Amft et al., 2015), reward circuitry (e.g., ventral striatum (VS), anterior insula (aIns), and dorsal mPFC; Haber and Knutson, 2010), and emotion regulation circuitry (e.g., ventrolateral PFC (vlPFC), anterior cingulate cortex (ACC); Steinberg, 2005) may play a role in adolescent sexual development. Studies in healthy adults demonstrate that viewing sexual stimuli indeed elicits activation of these regions independent of general emotional arousal (Wehrum et al., 2013; Wehrum-Osinsky et al., 2014), and greater vs responsiveness to sexual images predicts future sexual desire and "indulgence in" sexual behavior (Demos et al., 2012). Interactions among pubertal gonadal hormones are also postulated to impact development of these neural reward systems (for review, see Sinclair et al., 2014) and adolescent sexual behavior (for review, see Baams et al., 2015). Further, brain development and social experience can have a bidirectional influence. Based on non-human primate findings of hormonal changes in relation to sexual experience, it has been proposed that sexual behavior itself may exert an influence on adolescent brain development (Forbes and Dahl, 2010).

Despite the fact that all adolescents undergo development of these social and neural systems, engagement in risky behavior varies, with some far more likely than others to partake (Bjork and Pardini, 2015). One likely factor underlying individual differences in risk-taking behavior is function in social neurocircuitry (Victor and Hariri, 2016). Such differences may contribute to individual variability in motivation to experience the benefits of risky behavior, and in the extent to which the presence of peers affects risky decision-making (Albert et al., 2013). Notably, neural response to risky decisions is associated with future real-life engagement in such behavior (Crowley et al., 2015).

Consistent with proposed models involving heightened reward motivation and reactivity, higher tendency toward impulsivity is consistently linked to adolescents' risky sexual behavior (Dir et al., 2014), and greatervs activation has been observed in adults with compulsive sexual behaviors (Voon et al., 2014). Despite the likely involvement of neural reward circuitry in risky sexual behavior, the literature on its role in adolescent sexual risk-taking is scant. One recent study in young adults ages $18-22$ found that increasing ventral striatum activation during reward predicted the number of sexual partners (Victor et al., 2015). In contrast, the majority of available studies have examined inhibitory control, demonstrating a relationship between risky sexual behavior and weaker lateral prefrontal cortex response during response inhibition (Ewing et al., 2015; Goldenberg et al., 2013). No studies have examined functional connectivity in adolescent risky sexual behavior, which can reveal the network-level coordination of brain function during reward processing, nor have studies examined the influence of social context on sexual risk-associated neural activity.

In studying correlates of adolescent sexual behavior, it is critically important to distinguish between sexual behavior and sexual risk behavior. Research on adolescent sexual behavior has traditionally framed all sexual activity as risky and problematic, yet recent evidence indicates that sexual experience per se does not predict maladaptive outcomes-and may even be a marker of healthy social and psychological development (see Harden, 2014). In fact, recent conceptual models have emphasized the need for developmental neuroscience investigating sexual and romantic development as part of normative changes in social and affective circuitry (Suleiman et al., 2016). Evolutionary perspectives also highlight the normativity and centrality of sexual behavior during adolescence (Ellis et al., 2012). Although sexual behavior is a normative aspect of adolescent development, certain behaviors carry high levels of risk. Sexually active adolescents acquire half of all STIs (Center for Disease Control and Prevention, 2015) and an estimated $15 \%$ of sexually active adolescent girls aged 15-19 in the U.S. experience unintended pregnancy (Kost et al., 2010). Several specific types of behaviors are associated with increased risk and have been frequently studied in the adolescent sexual health literature, including a higher number of intercourse partners (Center for Disease Control and Prevention, 2015), early sexual debut (e.g., (DixonMueller, 2008)), the use of alcohol at last intercourse (Kann, 2016), and the absence of condoms or birth control at last intercourse (Kann, 2016).

Social reward, the experience arising from a positive social interaction (e.g., feeling accepted or being liked) is particularly salient during adolescence and activates neural reward and self-referential circuitry (Davey et al., 2010). Adolescents are also more likely to engage in risky behaviors during peer social interactions (Steinberg, 2008), which suggests that peer social reward is a relevant context for examining potential neural correlates or mechanisms of risky sexual behavior. As previous neuroimaging studies have examined aspects of regulatory, reward, and threat circuits in adolescent sexual risk-taking independent of social context, this study sought to examine neural function during the specific social context of social reward among adolescents engaging in higher-risk sexual behaviors. Using a social reward fMRI task, it was hypothesized that adolescents who engage in riskier sexual behaviors would exhibit heightened activation and greater connectivity within social reward circuits in response to being liked by peers.

\section{Methods}

\subsection{Participants}

Forty-seven adolescents ages 14-18 were included in the present study $(18 \mathrm{M}, 29 \mathrm{~F}$ [16.3 $\pm 1.4 \mathrm{yrs}] ; 67 \%$ Caucasian, $22 \%$ African American, $11 \% \mathrm{bi} / \mathrm{multi}$-racial). These individuals were recruited to participate in a study on social reward processing in typically developing late-to-post pubertal adolescents (Euling et al., 2008) with no history of psychiatric disorder, psychiatric treatment, or serious medical conditions. Individuals were recruited from the community using flyers in community settings, ads on Craigslist and other internet sources. 70 adolescents were enrolled in the study. Individuals were excluded from the final sample if they did not complete the fMRI scan due to scanning exclusionary criteria $(n=8 ; 3$ due to recent concussion, 3 due to claustrophobia, 2 due to mental health diagnosis), could not be contacted or withdrew from the study after their initial behavioral assessment $(n=5)$, did not complete the fMRI task or were removed due to scan quality $(n=8)$, or had missing behavioral data $(n=2)$. Male and female participants did not differ in age or race. The University of Pittsburgh Institutional Review Board approved all research procedures, and written informed consent was obtained from 
each participant and a parent or guardian.

\subsection{Measures}

\subsubsection{Sexual risk behavior}

The Youth Risk Behavior Survey 2009 (Eaton et al., 2011) is a validated epidemiologic self-report instrument developed for high school students and assessing health-risk behaviors, including sexual behaviors. Seven questions address sexual behavior, including ever having had sexual intercourse, age of first sexual intercourse, number of lifetime and past 3-month sexual intercourse partners, use of condoms at last sexual intercourse, use of pregnancy prevention method at last sexual intercourse, and use of alcohol at last sexual intercourse.

YRBS data were used to classify participants in two ways. First, two sexual risk groups were created: higher sexual risk $(n=16 ; 11 \mathrm{~F}, 5 \mathrm{M})$ and lower sexual risk $(n=31 ; 18 \mathrm{~F}, 13 \mathrm{M})$. Based on commonly used measures of sexual risk behavior (Kelley et al., 2003; Madkour et al., 2010; Center for Disease Control and Prevention, 2015), adolescents were classified as "higher sexual risk" if they reported any of the following: age of first sexual intercourse of 15 or younger, three or more lifetime intercourse partners, two or more intercourse partners in the past three months, lack of condom use at last intercourse, lack of pregnancy prevention method at last intercourse, or use of alcohol at last intercourse. Other adolescents were classified as "lower sexual risk." Note that the term "no sexual risk" was avoided for this lower-risk group of youth, as it was possible that these adolescents had engaged in sexual behaviors other than those assessed and that carry some degree of sexual risk (e.g., unprotected oral sex with multiple partners). However, the YRBS items were developed based on morbidity and mortality data on behavioral factors that contribute to HIV infection, other sexually transmitted infections, and unintended pregnancy (Center for Disease Control and Prevention, 2004).

Second, the "ever had sexual intercourse" variable was used in supplemental analyses that examined presence or absence of sexual intercourse experience (i.e., rather than solely focusing on sexual risk behavior). Twenty adolescents (13F, 7M) had engaged in sexual intercourse whereas 27 adolescents $(16 \mathrm{~F}, 11 \mathrm{M})$ did not have sexual intercourse experience. These analyses addressed the possible specificity of risky sexual experience rather than sexual experience per se in association with neural response to social reward.

\subsection{Non-sexual risk behavior}

To quantify general risk-taking behavior independent of sexual risktaking, a variable was computed based on a previously derived YRBS factor (Youssef et al., 2016), but excluding the variables in the original factor related to sexual risk taking. Specifically, a multiple correspondence analysis (MCA) was used in the current sample to reduce the categorical YRBS risk factors to a unidimensional non-sexual risk factor based on whether participants reported the following behaviors: bike helmet use, seat belt use, history of physical fighting, past suicide plan, daily smoking, days using alcohol, or lifetime use of marijuana or other illicit drugs (current study $\alpha=0.67$ ). This factor was used as a covariate in supplemental analyses to examine the specificity of findings to sexual risk behaviors.

\subsection{Social reward fMRI task}

All participants completed an fMRI social reward task to investigate neural response to positive social feedback (for details, see Healey et al., 2014). Briefly, prior to brain imaging (6.34 \pm 8.72 weeks), participants rated images of other adolescents ( 40 photos; $50 \%$ female) based on the degree to which they felt they would like the individuals ( 1 = "not at all"; 9 = "very much"). Participants were also informed that their own photos would be "rated" by the adolescents in the images. Participants' romantic or sexual attraction to the adolescents in the photos was not assessed. Personalized stimulus sets were created for each participant where photos were grouped according to ratings of the participant ostensibly provided by the peers in the photos. Specifically, each participant was shown that some peers in the photos had rated them favorably (positive feedback; photos presented on a green background), whereas others had not yet rated them (neutral feedback, or essentially no-feedback stimuli; photos presented on a white background).

Personalized stimulus sets were presented in a block design task composed of positive feedback and neutral feedback blocks. The 32 stimuli were each presented 3 times across the 8 blocks, with each block consisting of 12 stimuli lasting a total of 84s. Four of the blocks were positive feedback blocks and 4 were neutral feedback. To minimize habituation and predictability from the block design, each block contained two images of the opposite stimulus type (e.g. positive feedback blocks contained 10 positive feedback stimuli and 2 neutral feedback stimuli). Images were presented for $3 \mathrm{~s}$ with a jittered intertrial interval between stimuli and an interblock interval of 8 s. To confirm task engagement, participants were instructed to press a button every time a face appeared. Participants were debriefed about task deception after the scan and informed that their photo had not been shown to or rated by others.

\section{5. fMRI acquisition and preprocessing}

Structural and functional images were acquired using a Siemens 3.0 T TIM Trio scanner at the University of Pittsburgh Magnetic Resonance Research Center (MRRC). T1-weighted high-resolution MPRAGE structural images were acquired with $1 \mathrm{~mm}$ isometric voxels (TR/TE/flip angle $=2300 \mathrm{~ms} / 2.98 \mathrm{~ms} / 9 ; \mathrm{FOV}=256 \times 240 ; 1.2 \mathrm{~mm}$ slice; 160 slices; $256 \times 240$ matrix; $1 \mathrm{Nex}$ ). Functional blood oxygen level dependent (BOLD) images during the social reward task were acquired with a gradient echo planar imaging sequence and covered 39 axial slices, $3.1 \mathrm{~mm}$ thick, beginning at the cerebral vertex and encompassing the entire cerebrum and the majority of the cerebellum oriented to the AC-PC line (TR/TE $=2000 \mathrm{MS} / 30 \mathrm{~ms}$, FOV $=205 \times 205$, matrix $=64 \times 64$ ). All scanning parameters were selected to optimize the quality of the BOLD signal while maintaining a sufficient number of slices to acquire whole-brain data; therefore, prior to the collection of fMRI data for each participant, a reference EPI scan was acquired and inspected to confirm a good signal and the absence of artifacts across the entire volume of acquisition.

Preprocessing and fMRI image analysis was performed using SPM12 (http://www.fil.ion.ucl.ac.uk/spm). For each participant's scan, structural images were segmented and functional images were realigned, motion-corrected, and high-pass temporally filtered with a cutoff of $128 \mathrm{~s}$. Image volumes with high motion were adjusted using ART (http://gablab.mit.edu/index.php/software). The mean functional image was coregistered with the high-resolution 3D anatomic image, normalized to MNI space using a 12-parameter affine model, and spatially smoothed with a 6.0 FWHM Gaussian kernel.

\subsection{Data analysis}

Social reward was modeled in a first-level general linear model for each participant by contrasting the neural response during receipt of positive feedback to blocks with neutral feedback (all positive $>$ neutral feedback). Functional connectivity during social reward was measured during first-level analyses using generalized psychophysiological interaction (gPPI) in SPM12 (O'Reilly et al., 2012). Given our interest in how adolescents with higher risk sexual behavior respond during social situations, connectivity during the social reward task, as opposed to resting state functional connectivity, was examined. The precuneus was chosen as a seed region given its critical role in social reward and selfrelevant processing, as well as its robust activation in the current task (Healey et al., 2014), and was defined using WFU PickAtlas. 
Individual activation and functional connectivity contrast images were entered into second-level analyses to compare neural activation and connectivity in response to social reward between higher risk and lower risk groups. Given our focus on neural response to social reward as relevant to risky sexual behavior, a class of social behavior, results were constrained to social processing regions. This set of regions - including precuneus, bilateral temporoparietal junction, ventral striatum, anterior insula, medial and ventrolateral prefrontal cortex, and anterior cingulate cortex - was defined by the Social mask in Neurosynth, which is based on meta-analytic findings of fMRI studies using social paradigms ( $N=1000$ studies at the time of analyses; Yarkoni et al., 2011). In addition, whole-brain analyses were subsequently run to confirm the involvement of social regions and examine associations of risky sexual behavior with other brain regions. To address Type I error, Monte Carlo simulations were used to estimate the minimum number of contiguous

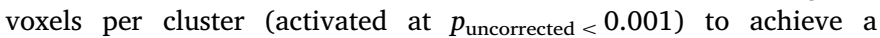
threshold of $p_{\text {corrected }}<0.05$. The resulting cluster extent threshold was 5 voxels for neural response and 16 voxels for functional connectivity analyses. Age and gender were included as covariates in all analyses considering their association with development of brain structure (Gogtay et al., 2004; Lenroot and Giedd, 2010) and function in neural reward circuity during adolescence (Smith et al., 2015).

\section{Results}

\subsection{Sexual behavior}

Sixteen individuals had engaged in one or more risky sexual behaviors and were classified into the higher-risk group. Individuals classified in this group had engaged in an average of $2.38 \pm 0.96$ sexual risk behaviors ( $n=3$ for 1 sexual risk behavior, $n=6$ for 2 behaviors, $n=5$ for 3 behaviors, and $n=2$ for 4 behaviors). Of those who had engaged in one sexual risk behavior, two were classified based on age of first sexual intercourse while the other was classified based on number of lifetime sexual partners. Two individuals who were classified as higher sexual risk had not used condoms at last sexual intercourse but were using pregnancy prevention, which could be considered low risk if both they and their partners were free of STIs. However, both participants also met criteria for additional sexual risk behaviors.

Adolescents in the higher-risk group were significantly more likely to engage in all individual sexual risk and non-sexual risk behaviors (see Table 1). Male and female participants did not differ in sexual risk behaviors with the exception of age of first intercourse. Although female participants overall had an earlier age of first sexual intercourse $(t$ $[45]=2.09, p=0.05$ ), within the higher and lower sexual risk groups, there were no differences between males and females in age of first intercourse $\left(\chi^{2}[1, N=47]=3.09, p=0.21\right)$. For these reasons, age and gender were included as covariates in all analyses.

\subsection{Risky sexual behavior and neural response to reward}

Compared with adolescents with lower-risk sexual behaviors, adolescents with higher-risk sexual behaviors demonstrated greater activation to social reward in the right precuneus and the right TPJ (see Table 2, Fig. 1). Adolescents with lower-risk sexual behaviors did not demonstrate any areas of greater activation. Whole brain analyses did not identify any additional regions of activation differing between higher and lower sexual risk adolescents in response to being liked. Gender did not moderate effects.

\subsection{Risky sexual behavior and precuneus connectivity during social reward}

In light of the heightened activation in the precuneus in response to social reward in adolescents with higher-risk sexual behaviors and the role of the precuneus in self and social processing (Rodrigo et al., 2014), group differences in functional connectivity between the precuneus and other social areas as defined by the Neurosynth social mask were examined. Individuals with higher-risk sexual behaviors demonstrated greater functional connectivity than those with lower-risk sexual behaviors between the precuneus and the TPJ bilaterally, as well as frontal regions, including one cluster involving the dorsal $\mathrm{mPFC}$ and rostral anterior cingulate cortex (rACC), and another involving the left vlPFC and anterior insula (see Table 3, Fig. 2). There were no regions for which the lower-risk group exhibited greater precuneus functional connectivity than the higher-risk group. Group differences in functional connectivity were not moderated by gender. Whole brain analyses did not reveal any additional areas in which high-risk and low-risk groups differed for precuneus functional connectivity.

\subsection{Potential confounds: sexual experience and general risk taking}

The initial analysis revealed that higher sexual risk individuals exhibited greater activation and precuneus connectivity during social reward. To determine whether results were specific to sexual risk behavior, rather than sexual experience per se, we conducted the main analyses a second time, comparing participants who had engaged in sexual intercourse with those who had not. All individuals in the higher sexual risk group had sexual intercourse experience, as did 4 individuals $(2 \mathrm{~F}, 2 \mathrm{M})$ in the lower sexual risk group. Thus, the sexual intercourse experience group contained the higher sexual risk individuals plus the 4 lower sexual risk adolescents with intercourse experience $(n=20 ; 13 \mathrm{~F}, 7 \mathrm{M})$. When participants were re-classified based on experience of sexual intercourse, groups with and without experience of sexual intercourse did not differ in neural activation or precuneus connectivity in response to social reward. This suggests that our findings on higher sexual risk behaviors could not be attributed simply to experience of sexual intercourse (see Supplementary Table 1).

Furthermore, to ensure that the 4 lower sexual risk adolescents with intercourse experience were not driving the original group difference results, we conducted the main analyses (higher vs. lower sexual risk groups) with these participants excluded. Results remained significant, with the exception that precuneus connectivity with the left TPJ was no longer greater among the higher sexual risk group. This suggests that these 4 participants were not driving the group difference (see Supplementary Table S1 for details).

Finally, to ensure that general risk taking was not influencing the results given that higher sexual risk individuals were also more likely to have engaged in non-sexual risk taking $(t[45]=-3.298, p=0.002)$, the initial activation and connectivity analyses for sexual risky-behavior groups were repeated using the non-sexual risk-taking factor as a covariate. The original group differences remained significant. Furthermore, correcting for non-sexual risk taking behavior strengthened the statistical significance for the majority of the functional connectivity results (see Supplementary Table S1).

These supplemental analyses indicated that the primary results were related to risky sexual behavior specifically, rather than to sexual intercourse experience or general risk-taking.

\section{Discussion}

In a sample of typically developing community adolescents, those who had engaged in higher risk sexual behaviors differed in neural response and functional connectivity to social reward. Adolescents with higher-risk sexual behavior had greater activation than adolescents with lower-risk sexual behavior in social reward-network regions including the precuneus and RTPJ, and heightened precuneus connectivity with social and affective processing regions. These differences appear to be specific to sexual risk taking, as engagement in other health-risk behaviors and mere sexual intercourse experience did not explain the results. Activation in these regions has preliminarily been associated more broadly with adolescent risk-taking during social situations (Rodrigo et al., 2014); however, this is the first study to 
Table 1

Subject Demographics and Sexual Behavior.

\begin{tabular}{|c|c|c|c|c|c|c|}
\hline & & \multicolumn{2}{|c|}{ Lower Sexual Risk } & \multicolumn{2}{|c|}{ Higher Sexual Risk } & \multirow[t]{2}{*}{ p-value } \\
\hline & & $\mathrm{N}$ & Mean \pm SD & $\mathrm{N}$ & Mean \pm SD & \\
\hline Age & & & $16.03 \pm 1.40 \mathrm{yrs}$ & & $16.93 \pm 1.16 y \mathrm{yrs}$ & 0.037 \\
\hline \multirow[t]{2}{*}{ Gender } & Female & 18 & $\mathrm{n} / \mathrm{a}$ & 5 & $\mathrm{n} / \mathrm{a}$ & 0.475 \\
\hline & Male & 13 & & 11 & & \\
\hline \multirow[t]{3}{*}{ Race } & White & 22 & $\mathrm{n} / \mathrm{a}$ & 10 & $\mathrm{n} / \mathrm{a}$ & 0.758 \\
\hline & Black & 6 & & 4 & & \\
\hline & Multiracial & 3 & & 2 & & \\
\hline \multirow[t]{6}{*}{ Parental Education } & Some high school & 0 & $\mathrm{n} / \mathrm{a}$ & 1 & $\mathrm{n} / \mathrm{a}$ & 0.307 \\
\hline & Completed high school & 3 & & 5 & & \\
\hline & Some college & 2 & & 1 & & \\
\hline & Completed college & 13 & & 4 & & \\
\hline & Technical degree & 3 & & 1 & & \\
\hline & Masters/Doctoral degree & 10 & & 4 & & \\
\hline \multirow[t]{2}{*}{ Sexual Intercourse } & No & 27 & $\mathrm{n} / \mathrm{a}$ & 0 & $\mathrm{n} / \mathrm{a}$ & $<0.001$ \\
\hline & Yes & 4 & & 16 & & \\
\hline \multirow[t]{3}{*}{ Alcohol Use During Last Sexual Intercourse } & No Sexual Partners & 27 & $\mathrm{n} / \mathrm{a}$ & 0 & $\mathrm{n} / \mathrm{a}$ & $<0.001$ \\
\hline & No & 4 & & 12 & & \\
\hline & Yes & 0 & & 4 & & \\
\hline \multirow[t]{3}{*}{ Condom Use During Last Sexual Intercourse } & No Sexual Partners & 27 & $\mathrm{n} / \mathrm{a}$ & 0 & $\mathrm{n} / \mathrm{a}$ & $<0.001$ \\
\hline & No & 0 & & 6 & & \\
\hline & Yes & 4 & & 10 & & \\
\hline \multirow[t]{3}{*}{ Pregnancy Prevention During Last Sexual Intercourse } & No Sexual Partners & 27 & $\mathrm{n} / \mathrm{a}$ & 0 & $\mathrm{n} / \mathrm{a}$ & $<0.001$ \\
\hline & No & 0 & & 4 & & \\
\hline & Yes & 4 & & 12 & & \\
\hline \multirow[t]{4}{*}{ Age of First Sexual Intercourse } & & $\mathrm{n} / \mathrm{a}$ & $16.75 \pm 0.05 y r s$ & $\mathrm{n} / \mathrm{a}$ & $15.00 \pm 1.26 \mathrm{yrs}$ & 0.016 \\
\hline & No Sexual Partners & 27 & $\mathrm{n} / \mathrm{a}$ & 0 & $\mathrm{n} / \mathrm{a}$ & $<0.001$ \\
\hline & 15 or younger & 0 & & 11 & & \\
\hline & 16 or older & 4 & & 5 & & \\
\hline \multirow[t]{4}{*}{ Lifetime Sexual Partners } & & $\mathrm{n} / \mathrm{a}$ & $0.13 \pm 0.34$ & $\mathrm{n} / \mathrm{a}$ & $3.25 \pm 1.77$ & $<0.001$ \\
\hline & No Sexual Partners & 27 & $\mathrm{n} / \mathrm{a}$ & 0 & $\mathrm{n} / \mathrm{a}$ & $<0.001$ \\
\hline & 1 or 2 Partners & 4 & & 6 & & \\
\hline & 3 or More Partners & 0 & & 10 & & \\
\hline \multirow[t]{4}{*}{ Three Month Sexual Partners } & & $\mathrm{n} / \mathrm{a}$ & $0.10 \pm 0.30$ & $\mathrm{n} / \mathrm{a}$ & $1.25 \pm 1.00$ & $<0.001$ \\
\hline & No Sexual Partners & 28 & $\mathrm{n} / \mathrm{a}$ & 2 & $\mathrm{n} / \mathrm{a}$ & $<0.001$ \\
\hline & 1 Sexual Partner & 3 & & 11 & & \\
\hline & 2 or More Partners & 0 & & 3 & & \\
\hline Non-Sexual Risk Taking (YRBS) & & $\mathrm{n} / \mathrm{a}$ & $-0.30 \pm 0.71$ & $\mathrm{n} / \mathrm{a}$ & $0.71 \pm 1.39$ & 0.002 \\
\hline
\end{tabular}

demonstrate heightened response of these socially relevant affective and reward processing circuits among adolescents who have engaged in risky sexual behavior specifically.

The TPJ, rACC, vlPFC, and dorsal mPFC are most often implicated as part of the default mode network, a set of regions whose coordinated function is postulated to underlie self-referential, reward, and social cognitive processing (Amft et al., 2015). The temporoparietal junction-particularly the right TPJ-is involved in reorienting towards and processing socially-relevant information (Krall et al., 2015). The anterior insula and vlPFC are important regions for reward salience and affect regulation, respectively (Smith et al., 2014; Steinberg, 2008). Notably, brain activation in these regions has been associated with broader risky decision making following peer evaluation (Falk et al., 2014; Peake et al., 2013). Dorsal mPFC and rACC have functional and structural connectivity with the ventral striatum (Haber et al., 2006; Haber and Knutson, 2010) and are thought to play a modulatory role in reward processing (Goto and Grace, 2008). Dorsomedial PFC is also putatively associated with both intrinsic reward and social processing (de la Vega et al., 2016). Thus, while heightened vs activation or functional connectivity with the precuneus was not observed specifically among higher sexual risk adolescents, the vs may still be playing a role in riskier sexual behavior through modulation of frontal regions.

The observed functional coordination among social, reward, and affective-processing regions among higher sexual risk adolescents likely reflects a complex interplay among several factors. These could include adolescents' individuals' sexual experiences, tendencies toward interpersonal reward seeking and enjoyment, sensitivity to socially rewarding stimuli, and orientation toward social rewards. The use of the precuneus, a region implicated in autobiographical memory, self, and agency (Krall et al., 2015) as functional connectivity seed indicates that circuitry mediating self-relevant and social behavior is altered in adolescents who have had experiences more commonly associated with STIs and other adverse health outcomes (Ashenhurst et al., 2017). Whereas previous studies have focused on immature executive function as a key factor in adolescent risky sexual decision making and regional brain responsiveness (Ewing et al., 2015; Goldenberg et al., 2013), our

Table 2

Increased activation in response to being liked among adolescents with higher sexual risk.

\begin{tabular}{|c|c|c|c|c|c|c|c|}
\hline Region & Cluster p-value & Cluster Size & Peak p-value (FWE correction) & T-Score & $\mathrm{X}$ & $\mathrm{Y}$ & $\mathrm{Z}$ \\
\hline \multicolumn{8}{|c|}{ Higher sexual risk $>$ Lower sexual risk } \\
\hline Right Precuneus & 0.016 & 46 & 0.096 & 4.45 & 8 & -60 & 36 \\
\hline Right Temporoparietal Junction & 0.031 & 15 & 0.373 & 3.88 & 48 & -52 & 40 \\
\hline \multicolumn{8}{|c|}{ Lower sexual risk $>$ Higher sexual risk } \\
\hline No regions of significance & & & & & & & \\
\hline
\end{tabular}




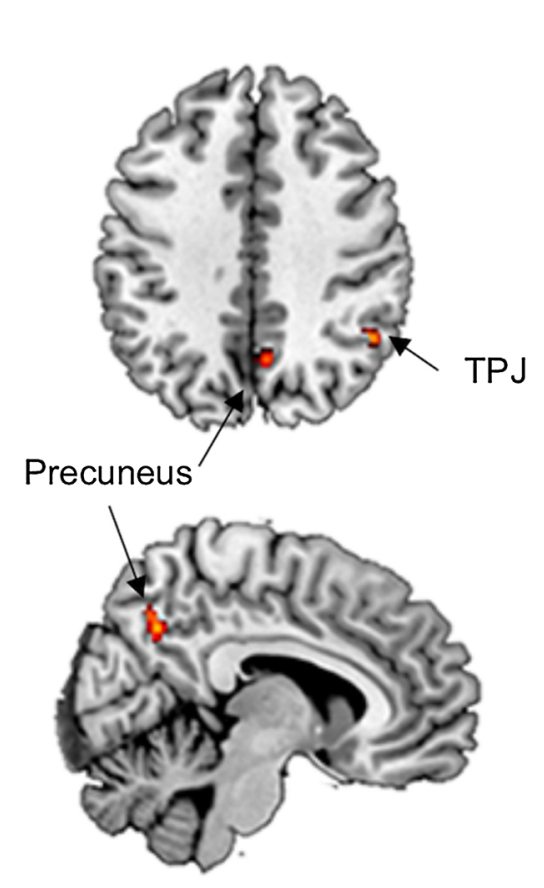

TPJ

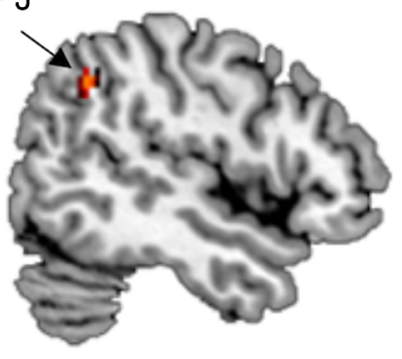

3.29

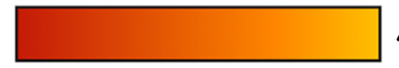

4.39

T-Score

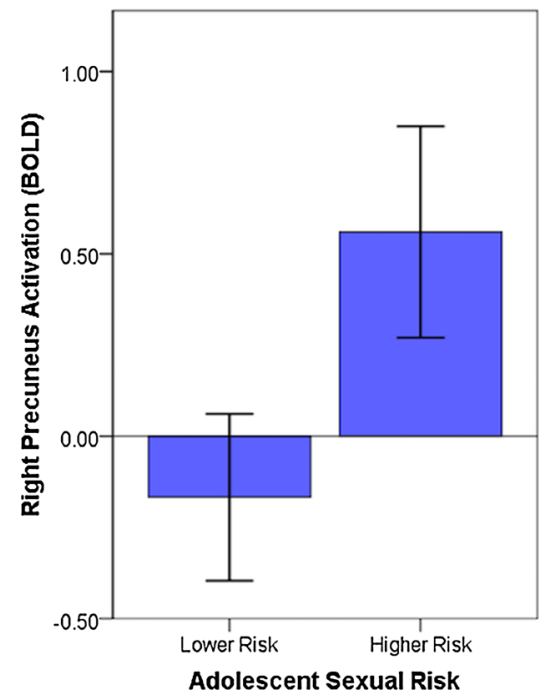

Fig. 1. Increased precuneus and right temporoparietal junction activation in response to being liked in adolescents with higher risk sexual behavior. TPJ; temporoparietal function.

Table 3

Increased precuneus functional connectivity with social reward regions in response to being liked among adolescents with higher sexual risk.

\begin{tabular}{|c|c|c|c|c|c|c|c|}
\hline Region & Cluster p-value & Cluster Size & Peak p-value (FWE correction) & T-Score & $\mathrm{X}$ & $\mathrm{Y}$ & $\mathrm{Z}$ \\
\hline \multicolumn{8}{|l|}{ Higher sexual risk $>$ Lower sexual risk } \\
\hline \multirow[t]{2}{*}{ Right Temporoparietal Junction } & 0.002 & 121 & 0.023 & 5.01 & 50 & -64 & 8 \\
\hline & 0.023 & 42 & 0.265 & 4.07 & 48 & -50 & 6 \\
\hline Dorsal Medial Prefrontal Cortex/Rostral ACC & 0.004 & 94 & 0.027 & 4.94 & 4 & 58 & -6 \\
\hline Left Ventrolateral Prefrontal Cortex/Anterior Insula & 0.025 & 39 & 0.361 & 3.99 & -36 & 16 & -14 \\
\hline Left Temporoparietal Junction & 0.035 & 27 & 0.311 & 3.92 & -58 & -60 & 10 \\
\hline \multicolumn{8}{|l|}{ Lower sexual risk $>$ Higher sexual risk } \\
\hline No regions of significance & & & & & & & \\
\hline
\end{tabular}

results emphasize the contributing role of function in social and affective circuitry (Victor and Hariri, 2016). Indeed, experts from the fields of adolescent sexual health (e.g., Halpern, 2010) and developmental neuroscience (e.g., Victor and Hariri, 2016) have highlighted the need for research that considers both the social and biological contexts of adolescent sexual behavior. Adolescence is a developmental period marked by a notable increase in neural and biological sensitivity to social reward, which contributes to greater engagement in general risktaking behaviors (Gardner and Steinberg, 2005; Knoll et al., 2015). Yet the intersecting social and biological influences on adolescent risk behavior may be especially relevant for sexual risk behavior.
In the current study, differences in brain activity and precuneus functional connectivity were observed based on adolescents' level of sexual risk behavior specifically, rather than their general engagement in risky behavior. This finding may be due to several unique aspects of adolescent sexual risk behavior. Specifically, sexual risk-taking is inherently interpersonal and influenced by a powerful combination of heightened interoceptive physiologic experiences (e.g. sexual arousal), personal traits (e.g. sensation-seeking), social goals (e.g. motivation for an intimate connection), and environmental cues (e.g. the presence of a potential sexual partner) which in combination may overwhelm adolescents' still-developing capacity for behavioral control (Victor and 


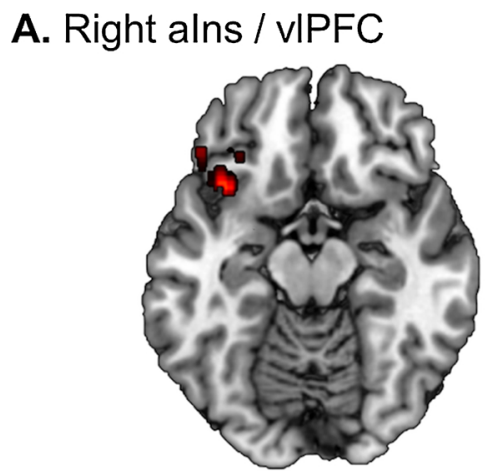

\section{B. Dorsal mPFC / rACC}
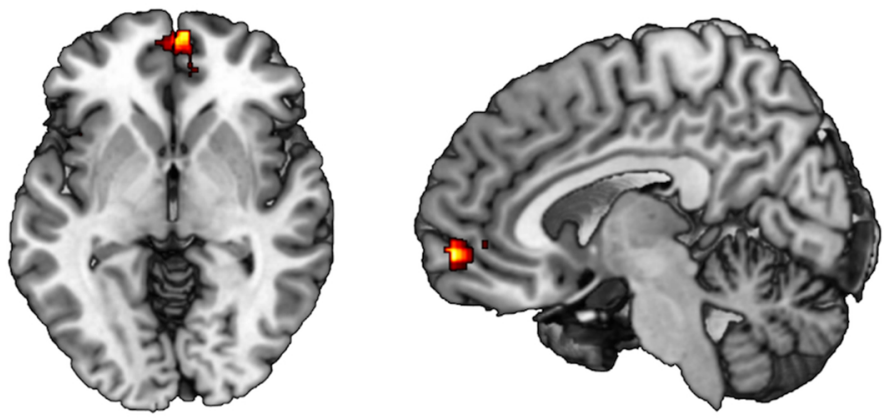

C. Left TPJ

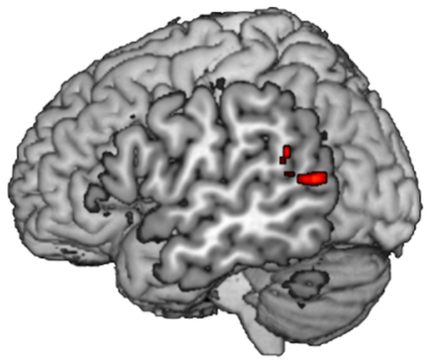

D. Right TPJ

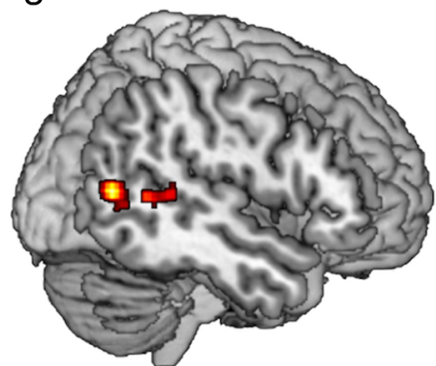

3.29

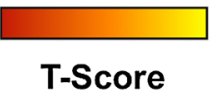

5.01

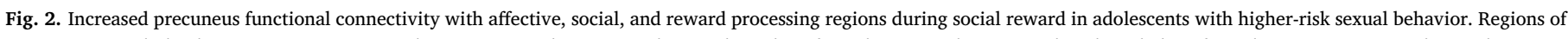

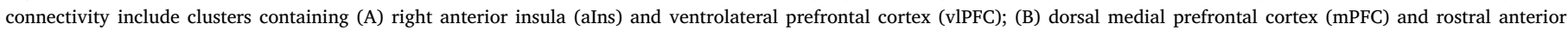
cingulate cortex (rACC); (C) left temporoparietal junction (TPJ), and (D) right temporoparietal junction.

Hariri, 2016). The current findings underscore the importance of considering the intersection of interpersonal and biological factors in adolescents' sexual risk behavior.

The association between brain and behavior is likely to be bidirectional, and the present results should be interpreted with the understanding that a specific pattern of neural response to social reward is likely not a causal mechanism for risky sexual behavior. That is, heightened response to social reward does not necessarily motivate risky sexual behavior. However, one could speculate that the factors underlying the neural response among higher sexual risk youth, including sensitivity to peer feedback or sexual desire arising from peer attractiveness, influence the function of reward and affective neural circuits, such that sexual risk evaluation is biased towards immediate sexual rewards over long-term health. As non-human primate studies have indicated, brain function changes as a result of sexual experiences and contact (Olsen, 2011; Staffend et al., 2014). The effects of sexual experience could have both adaptive and maladaptive consequences at many levels, including function in endocrine systems, development of brain structure, and sensitivity of brain function. For example, reward from engaging in less-risky romantic and/or sexual activity may be advantageous in developing the ability to make safer decisions, develop intimacy in relationships, and establish a positive sexual identity (for reviews, see Harden, 2014; Tolman and McClelland, 2011). Indeed, adolescents' engagement in sexual intercourse per se does not increase the risk of maladaptive outcomes (see Harden, 2014). However, the neurodevelopmental and behavioral balance between typically developing and safe experiences versus risky experiences remains unclear and underscores the critical need for research distinguishing between sexual risk behavior and broader sexual behavior. Further neurodevelopmental research aimed at understanding differences in the motivation for, initiation and practice of healthy sexual behavior-as well as their neural mechanisms-is essential for understanding and promoting adolescent health, including sexual health.

The involvement of reward and socioaffective processing regions with risky sexual experience provides support for promising treatment interventions. Studies of adolescents engaging in intervention programs promoting sexual health have demonstrated that interventions utilizing an interpersonal approach have been shown to reduce risk behaviors (Johnson et al., 2011; Petrova and Garcia-Retamero, 2015). Successful interpersonal interventions, including those that focus on skills such as assertiveness, refusal strategies based on personal sexual values, negotiation, and effective parent-youth communication, are often implemented in a social context with peers or parents (Fish et al., 2014; Jemmott et al., 1999; Rolleri et al., 2011). The present results underscore the importance of practicing skill-based strategies supporting healthy sexual behavior in a positive (e.g. rewarding) social environment. Given that culture and community are important aspects of social context, it is notable that these programs have particular benefit when being tailored towards specific communities (Goesling et al., 2014).

One clear limitation to the present study is the sample size. As a result, while conventional thresholds and covariates were used in statistical models to correct for Type I error, there remains a chance for Type II error, or the chance that analyses were underpowered to detect smaller but meaningful group differences. Another limitation of the present study is that adolescents were classified as higher and lower sexual risk based on their behaviors related to sexual intercourse. It is possible that adolescents in the "lower sexual risk" category, the majority of whom had not engaged in sexual intercourse, had in fact engaged in other behaviors that carry risk but are not assessed by the YRBS, such as unprotected oral sex with multiple partners or sex under the influence of non-alcoholic substances. Similarly, there was clearly variation in degree of sexual risk among the higher sexual risk individuals that was not reflected in the binary classification of participants as of higher and lower sexual risk. However, the specific sexual risk behaviors were operationalized through a validated risk survey (Eaton et al., 2011) and have been associated with HIV/STIs, unplanned pregnancy, and likelihood of engaging in future risky sexual behavior, and they therefore represent an important component of higher sexual risk (Ashenhurst et al., 2017). The lower sexual risk group was younger than the higher sexual risk group, given that the participants who had not had sex (e.g. lower sexual risk) were unsurprisingly younger than those who had. It could be argued that the individuals who have not 
had sexual intercourse were younger and may engage in sexual risk behaviors in the future. While these individuals are currently lower risk based on choosing to abstain from sexual intercourse and all results were corrected for age, additional research is necessary to determine the exact factors and neural circuits underlying the transition from lower to higher risk behaviors. Future studies should examine a wider range of sexual behaviors with greater information on context of risk, as well as the relationship between the neural correlates of risky sexual behavior and the aforementioned outcomes associated with sexual risktaking prospectively. Lastly, the composition and attractiveness of the photo stimuli are important to consider. Participants were only asked to rate their liking of the individual in the photo, not their physical attraction to the person in the photo. Romantic or sexual attraction could potentially have affected participants' original rating of stimuli and, subsequently, their personalized stimulus set. While significant effects for neural activation and functional connectivity were revealed by a task presenting social reward from unfamiliar peers, a majority of research examining peer influence on risky sexual behaviors has focused on familiar peers (Buhi and Goodson, 2007; Choukas-Bradley et al., 2014). Future work should examine neural activation and connectivity in connection with known peers-including romantic partners-and the implications for sexual risk behaviors.

\section{Conclusion}

Sexual behavior is a key part of adolescent development that is understudied in the behavioral literature, not to mention in the developmental neuroscience literature. The present study demonstrated that adolescents engaging in higher risk sexual behaviors exhibited greater neural activation and functional connectivity in social and affective processing regions during social reward compared with those with lower sexual risk behaviors. These results withstood adjustment for experience with sexual intercourse and engagement in other, nonsexual, risk behaviors. The findings, which importantly distinguish sexual risk behaviors from broader sexual experience and general risk taking, elucidate some of the neural mechanisms in the development of risky sexual behavior and emphasize the importance of both social and biological contexts in the development of adolescent sexual behavior.

\section{Funding}

This work was supported by National Institutes of Health grant R21 DA033612 (E Forbes, PI).

\section{Conflict of Interest}

None.

\section{Appendix A. Supplementary data}

Supplementary data associated with this article can be found, in the online version, at http://dx.doi.org/10.1016/j.dcn.2017.07.004.

\section{References}

Abma, J.C., Martinez, G.M., Copen, C.E., 2010. Teenagers in the United States: sexual activity, contraceptive use, and childbearing, national survey of family growth 2006-2008. Vital Health Stat. S23, Data from the National Survey of Family Growth 30, 1-47.

Albert, D., Chein, J., Steinberg, L., 2013. The teenage brain: peer influences on adolescent decision making. Curr. Dir. Psychol. Sci. 22 (2), 114-120.

Amft, M., Bzdok, D., Laird, A.R., Fox, P.T., Schilbach, L., Eickhoff, S.B., 2015. Definition and characterization of an extended social-affective default network. Brain Struct. Funct. 220 (2), 1031-1049.

Ashenhurst, J.R., Wilhite, E.R., Harden, K.P., Fromme, K., 2017. Number of sexual partners and relationship status are associated with unprotected sex across emerging adulthood. Arch. Sex. Behav. 46 (2), 419-432.

Baams, L., Dubas, J.S., Overbeek, G., Van Aken, M.A., 2015. Transitions in body and behavior: a meta-analytic study on the relationship between pubertal development and adolescent sexual behavior. J. Adolesc. Health 56 (6), 586-598.

Bjork, J.M., Pardini, D.A., 2015. Who are those risk-taking adolescents? Individual differences in developmental neuroimaging research. Dev. Cognitive Neurosci. 11, 56-64.

Brechwald, W.A., Prinstein, M.J., 2011. Beyond homophily: a decade of advances in understanding peer influence processes. J. Res. Adolesc. 21 (1), 166-179.

Buhi, E.R., Goodson, P., 2007. Predictors of adolescent sexual behavior and intention: a theory-guided systematic review. J. Adolesc. Health 40 (1), 4-21.

Casey, B.J., Jones, R.M., Hare, T.A., 2008. The adolescent brain. Ann. N. Y. Acad. Sci. 1124 (1), 111-126.

Center for Disease Control and Prevention, 2004. Methodology of the Youth Risk Behavior Surveillance System. Morbidity and Mortality Weekly Report. pp. 53.

Center for Disease Control and Prevention, 2015. Sexually Transmitted Disease Surveillance. http://www.cdc.gov/std/stats14/surv-2014-print.pdf.

Choukas-Bradley, S., Giletta, M., Widman, L., Cohen, G.L., Prinstein, M.J., 2014 Experimentally measured susceptibility to peer influence and adolescent sexual behavior trajectories: a preliminary study. Dev. Psychol. 50 (9), 2221-2227.

Crone, E.A., Dahl, R.E., 2012. Understanding adolescence as a period of social-affective engagement and goal flexibility. Nat. Rev. Neurosci. 13 (9), 636-650.

Crowley, T.J., Dalwani, M.S., Mikulich-Gilbertson, S.K., Young, S.E., Sakai, J.T., Raymond, K.M., Banich, M.T., 2015. Adolescents' neural processing of risky decisions: effects of sex and behavioral disinhibition. PLoS One 10 (7), e0132322.

Davey, C.G., Allen, N.B., Harrison, B.J., Dwyer, D.B., Yücel, M., 2010. Being liked activates primary reward and midline self-related brain regions. Hum. Brain Mapp. 31 (4), 660-668.

Demos, K.E., Heatherton, T.F., Kelley, W.M., 2012. Individual differences in nucleus accumbens activity to food and sexual images predict weight gain and sexual behavior. J. Neurosci. 32 (16), 5549-5552.

de la Vega, A., Chang, L.J., Banich, M.T., Wager, T.D., Yarkoni, T., 2016. Large-scale meta-analysis of human medial frontal cortex reveals tripartite functional organization. J. Neurosci. 36 (24), 6553-6562.

Dir, A.L., Coskunpinar, A., Cyders, M.A., 2014. A meta-analytic review of the relationship between adolescent risky sexual behavior and impulsivity across gender, age, and race. Clin. Psychol. Rev. 34 (7), 551-562.

Dixon-Mueller, R., 2008. How young is too young? Comparative perspectives on adolescent sexual, marital, and reproductive transitions. Stud. Fam. Plann. 39 (4), $247-262$.

Eaton, D.K., Kann, L., Kinchen, S., Shanklin, S., Ross, J., Hawkins, J., Chyen, D., 2011. Youth Risk Behavior Surveillance-United States, 2009 Morbidity and Mortality Weekly Report. pp. 59.

Ellis, B.J., Del Giudice, M., Dishion, T.J., Figueredo, A.J., Gray, P., Griskevicius, V., Volk, A.A., 2012. The evolutionary basis of risky adolescent behavior: implications for science, policy, and practice. Dev. Psychol. 48 (3), 598.

Ernst, M., Fudge, J.L., 2009. A developmental neurobiological model of motivated behavior: anatomy, connectivity and ontogeny of the triadic nodes. Neurosci. Biobehav. Rev. 33 (3), 367-382.

Euling, S.Y., Herman-Giddens, M.E., Lee, P.A., Selevan, S.G., Juul, A., SØrensen, T.I.A., Dunkel, L., Himes, J.H., Tielmann, G., Swan, S.H., 2008. Examination of US pubertytiming data from 1940 to 1994 for secular trends: panel findings. Pediatrics 121, S172-S191.

Ewing, S.W.F., Houck, J.M., Bryan, A.D., 2015. Neural activation during response inhibition is associated with adolescents' frequency of risky sex and substance use. Addict. Behav. 44, 80-87.

Falk, E.B., Cascio, C.N., O’Donnell, M.B., Carp, J., Tinney, F.J., Bingham, C.R., SimonsMorton, B.G., 2014. Neural responses to exclusion predict susceptibility to social influence. J. Adolesc. Health 54 (5), S22-S31.

Fish, H., Manlove, J., Moore, K.A., Mass, E., 2014. What works for adolescent sexual and reproductive health: lessons from experimental evaluations of programs and interventions. Child Trends Available at: https://www.childtrends.org/wp-content/ uploads/2015/08/2014-64WhatWorksTeenSexualReproHealth2.pdf.

Forbes, E.E., Dahl, R.E., 2010. Pubertal development and behavior: hormonal activation of social and motivational tendencies. Brain Cogn. 72 (1), 66-72.

Gardner, M., Steinberg, L., 2005. Peer influence on risk taking, risk preference, and risky decision making in adolescence and adulthood: an experimental study. Dev. Psychol. 41 (4), 625.

Goesling, B., Colman, S., Trenholm, C., Terzian, M., Moore, K., 2014. Programs to reduce teen pregnancy, sexually transmitted infections, and associated sexual risk behaviors: a systematic review. J. Adolesc. Health 54 (5), 499-507.

Gogtay, N., Giedd, J.N., Lusk, L., Hayashi, K.M., Greenstein, D., Vaituzis, A.C., Thompson, P.M., 2004. Dynamic mapping of human cortical development during childhood through early adulthood. Proc. Natl. Acad. Sci. U. S. A. 101 (21), 8174-8179.

Goldenberg, D., Telzer, E.H., Lieberman, M.D., Fuligni, A., Galván, A., 2013. Neural mechanisms of impulse control in sexually risky adolescents. Dev. Cognitive Neurosci. 6, 23-29.

Gomes, F.V., Rincón-Cortés, M., Grace, A.A., 2016. Adolescence as a period of vulnerability and intervention in schizophrenia: insights from the MAM Model. Neurosci. Biobehav. Rev. 70, 260-270.

Goto, Y., Grace, A.A., 2008. Limbic and cortical information processing in the nucleus accumbens. Trends Neurosci. 31 (11), 552-558.

Haber, S.N., Knutson, B., 2010. The reward circuit: linking primate anatomy and human imaging. Neuropsychopharmacology 35 (1), 4-26.

Haber, S.N., Kim, K.-S., Mailly, P., Calzavara, R., 2006. Reward-related cortical inputs define a large striatal region in primates that interface with associative cortical connections, providing a substrate for incentive-based learning. J. Neurosci. 26 (32), 8368-8376.

Halpern, C.T., 2010. Reframing research on adolescent sexuality: healthy sexual 
development as part of the life course. Perspect. Sex Reprod. Health 42 (1), 6-7.

Harden, K.P., 2014. A sex-positive framework for research on adolescent sexuality. Perspect. Psychol. Sci. 9 (5), 455-469.

Healey, K.L., Morgan, J., Musselman, S.C., Olino, T.M., Forbes, E.E., 2014. Social anhedonia and medial prefrontal response to mutual liking in late adolescents. Brain Cogn. 89, 39-50.

Jemmott, J.B., Jemmott, L.S., Fong, G.T., McCaffree, K., 1999. Reducing HIV risk-associated sexual behavior among African American adolescents: testing the generality of intervention effects. Am. J. Community Psychol. 27 (2), 161-187.

Johnson, B.T., Scott-Sheldon, L.A., Huedo-Medina, T.B., Carey, M.P., 2011. Interventions to reduce sexual risk for human immunodeficiency virus in adolescents: a metaanalysis of trials, 1985-2008. Arch. Pediatr. Adolesc. Med. 165 (1), 77-84.

Kann, L., 2016. Youth risk behavior Surveillance-United States, 2015. MMWR Surveill. Summ. 65.

Kelley, S.S., Borawski, E.A., Flocke, S.A., Keen, K.J., 2003. The role of sequential and concurrent sexual relationships in the risk of sexually transmitted diseases among adolescents. J. Adolesc. Health 32 (4), 296-305.

Knoll, L.J., Magis-Weinberg, L., Speekenbrink, M., Blakemore, S.J., 2015. Social influence on risk perception during adolescence. Psychol. Sci. 26 (5), 583-592.

Kost, K., Henshaw, S., Carlin, L., 2010. US Teenage Pregnancies, Births and Abortions: National and State Trends and Trends by Race and Ethnicity. Guttmacher Institute, NY Available at: https://www.guttmacher.org/sites/default/files/report_pdf/ ustptrends10.pdf.

Krall, S., Rottschy, C., Oberwelland, E., Bzdok, D., Fox, P., Eickhoff, S., Konrad, K., 2015. The role of the right temporoparietal junction in attention and social interaction as revealed by ALE meta-analysis. Brain Struct. Funct. 220 (2), 587-604.

Lenroot, R.K., Giedd, J.N., 2010. Sex differences in the adolescent brain. Brain Cogn. 72 (1), 46-55.

Luna, B., Marek, S., Larsen, B., Tervo-Clemmens, B., Chahal, R., 2015. An integrative model of the maturation of cognitive control. Annu. Rev. Neurosci. 38, 151-170.

Madkour, A.S., Farhat, T., Halpern, C.T., Godeau, E., Gabhainn, S.N., 2010. Early adolescent sexual initiation as a problem behavior: a comparative study of five nations. J. Adolesc. Health 47 (4), 389-398.

Mayeux, L., Sandstrom, M.J., Cillessen, A.H., 2008. Is being popular a risky proposition? J. Res. Adolesc. 18 (1), 49-74.

O’Reilly, J.X., Woolrich, M.W., Behrens, T.E., Smith, S.M., Johansen-Berg, H., 2012. Tools of the trade: psychophysiological interactions and functional connectivity. Soc. Cogn. Affect. Neurosci. 7 (5), 604-609.

Olsen, C.M., 2011. Natural rewards, neuroplasticity, and non-drug addictions. Neuropharmacology 61 (7), 1109-1122.

Peake, S.J., Dishion, T.J., Stormshak, E.A., Moore, W.E., Pfeifer, J.H., 2013. Risk-taking and social exclusion in adolescence: neural mechanisms underlying peer influences on decision-making. Neuroimage 82, 23-34.

Petrova, D., Garcia-Retamero, R., 2015. Effective evidence-based programs for preventing sexually-transmitted infections: a meta-analysis. Curr. HIV Res. 13 (5), 432-438.

Rodrigo, M.J., Padrón, I., De Vega, M., Ferstl, E.C., 2014. Adolescents' risky decisionmaking activates neural networks related to social cognition and cognitive control processes. Front. Hum. Neurosci. 8 (60) (eCollection 2014).
Rolleri, L.A., Lezin, N., Taylor, J., Moore, C., Wilson, M.M., Fuller, T., Firpo-Triplett, R., St. Lawrence, J., 2011. Becoming a Responsible Teen Adaptation Kit. ETR Associates, Santa Cruz, CA.

Sebastian, C., Burnett, S., Blakemore, S.-J., 2008. Development of the self-concept during adolescence. Trends Cogn. Sci. 12 (11), 441-446.

Sinclair, D., Purves-Tyson, T.D., Allen, K.M., Weickert, C.S., 2014. Impacts of stress and sex hormones on dopamine neurotransmission in the adolescent brain. Psychopharmacology (Berl.) 231 (8), 1581-1599.

Smith, A.R., Steinberg, L., Chein, J., 2014. The role of the anterior insula in adolescent decision making. Dev. Neurosci. 36 (3-4), 196-209.

Smith, A.R., Steinberg, L., Strang, N., Chein, J., 2015. Age differences in the impact of peers on adolescents' and adults' neural response to reward. Dev. Cognit. Neurosci. $11,75-82$.

Somerville, L.H., Jones, R.M., Casey, B., 2010. A time of change: behavioral and neural correlates of adolescent sensitivity to appetitive and aversive environmental cues. Brain Cogn. 72 (1), 124-133.

Staffend, N.A., Hedges, V.L., Chemel, B.R., Watts, V.J., Meisel, R.L., 2014. Cell-type specific increases in female hamster nucleus accumbens spine density following female sexual experience. Brain Struct. Funct. 219 (6), 2071-2081.

Steinberg, L., 2005. Cognitive and affective development in adolescence. Trends Cogn. Sci. 9 (2), 69-74.

Steinberg, L., 2008. A social neuroscience perspective on adolescent risk-taking. Dev. Rev. 28 (1), 78-106.

Tolman, D.L., McClelland, S.I., 2011. Normative sexuality development in adolescence: a decade in review, 2000-2009. J. Res. Adolesc. 21 (1), 242-255.

Victor, E.C., Hariri, A.R., 2016. A neuroscience perspective on sexual risk behavior in adolescence and emerging adulthood. Dev. Psychopathol. 28 (02), 471-487.

Victor, E.C., Sansosti, A.A., Bowman, H.C., Hariri, A.R., 2015. Differential patterns of amygdala and ventral striatum activation predict gender-specific changes in sexual risk behavior. J. Neurosci. 35 (23), 8896-8900.

Voon, V., Mole, T.B., Banca, P., Porter, L., Morris, L., Mitchell, S., 2014. Neural correlates of sexual cue reactivity in individuals with and without compulsive sexual behaviours. PLoS One 9, e102419.

Wehrum, S., Klucken, T., Kagerer, S., Walter, B., Hermann, A., Vaitl, D., Stark, R., 2013. Gender commonalities and differences in the neural processing of visual sexual stimuli? J. Sex. Med. 10 (5), 1328-1342.

Wehrum-Osinsky, S., Klucken, T., Kagerer, S., Walter, B., Hermann, A., Stark, R., 2014. At the second glance: stability of neural responses toward visual sexual stimuli. J. Sex. Med. 11, 2720-2737.

Williams, L.R., Russell, S.T., 2013. Shared social and emotional activities within adolescent romantic and non-romantic sexual relationships. Arch. Sex. Behav. 42 (4), 649-658.

Yarkoni, T., Poldrack, R.A., Nichols, T.E., Van Essen, D.C., Wager, T.D., 2011. Large-scale automated synthesis of human functional neuroimaging data. Nat. Methods 8 (8), 665-670.

Youssef, G.J., Whittle, S., Allen, N.B., Lubman, D.I., Simmons, J.G., Yucel, M., 2016. Cognitive control as a moderator of temperamental motivations toward adolescent risk-taking behavior. Child Dev. 87 (2), 395-404. 